\title{
Deep Drilling of Structural Timber with High Power Ultrashort Pulsed Laser Radiation
}

\author{
Benedikt Bornschlegel $^{* 1}$, Dennis Haasler ${ }^{1}$, Johannes Finger ${ }^{2}$, Denis Grizmann ${ }^{3}$, \\ Martin Trautz ${ }^{3}$ and Arnold Gillner ${ }^{1,2}$ \\ ${ }^{1}$ Chair for Laser Technology LLT, RWTH Aachen University \\ Steinbachstr. 15, 52074 Aachen, Germany \\ ${ }^{2}$ Fraunhofer Institute for Laser Technology ILT \\ Steinbachstr. 15, 52074 Aachen, Germany \\ ${ }^{3}$ Chair of Structures and Structural Design TRAKO, RWTH Aachen University \\ Schinkelstraße 1, 52062 Aachen, Germany \\ *Corresponding author's e-mail: benedikt.bornschlegel@,llt.rwth-aachen.de
}

\begin{abstract}
Deep drilling of structural timber using ultra short pulse laser ablation is investigated directed towards using this for a novel approach for reinforcement and connection of structural timber. To gain a basic understanding of the interaction processes, the optical properties as well as the different ablation behavior of early and late wood are investigated and discussed. Based on these findings a hypothesis is formulated that directly links the local cell density with the ablation behavior of wood. Furthermore, deep drilling processes are successfully applied to structural timber despite inhomogeneities of the material. We show that the damaging effects of the laser drilling on the mechanical properties of the realized screw connections is negligible.
\end{abstract}

DOI: $10.2961 /$ jlmn.2020.03.2007

Keywords: ultrashort pulsed, structural timber, wood, glulam, USP, ablation, deep drilling, engraving

\section{Introduction}

Machining wood by means of laser radiation has a long history. First attempts to cut wood with CO2-laser sources were already carried out in the late sixties and early seventies $[1,2]$ and led to processes which are established in nowadays industrial applications. Those include the furniture [3] and packaging industry, precision mechanics and model making. The advantages of processing wood by means of laser radiation compared with conventional machining processes (e.g. sawing, milling, knife planning and so on) are versatile. The key benefits are: no force is exerted onto the workpiece, there is no tool wear, unlimited complexity of the desired form and the kerfs are narrow [1]. Furthermore, laser processes are considering the current trend of individualizing products and digitalization of industrial production lines [4].

Besides the cutting of wood with CO2-laser, a special type of impregnation of wood with this laser sources were investigated. Small holes or cuts are created in the wood to increase the liquid uptake/infusion of the impregnation liquid into the wood material [5-9].

Since the ablation of wood with a continuous wave $(\mathrm{CW})$ or quasi-CW CO2-laser is a thermal process [10], the thermal degeneration of the material and the heat affected zone must be considered for all applications. Due to the carbonization of the machined surface, those processes can only be applied for products where carbonized cutting edges are irrelevant or the carbonization is required for marking and engraving [3]. However, if carbonization has to be avoided for optical or functional reasons, shorter pulsed laser sources are necessary. Therefore, the use of nanosecond (ns) pulsed laser sources with various wavelengths was investigated for the ablation of wood [11-13]. All applied ns-lasers sources can successfully machine wood regardless of a wavelength dependency being evident [12], nonetheless a degeneration such as carbonization of the surface cannot be avoided completely $[12,14]$. Only ultrashort pulsed (USP) lasers sources are capable of ablating wood with negligible heat input [14] into the work piece. Therefore, no carbonization occurs [15] and even the surface cell structures remain undamaged [16]. Likewise, micromechanical samples for tensile- and compression strength tests were successfully machined by applying a USP laser source [17]. Accordingly, modern high power USP laser systems can possibly be utilized to achieve efficient processing on a macroscopic scale with negligible thermal degradation of the material.

One of those new possible application is the drilling of deep guide holes for long screws. The need arises from the field of timber engineering where recently new approaches emerged. Such approaches utilize long continuously threaded self-tapping screws (STS) for reinforcements and connection of glued laminated timber $[18,19]$. An example of an application using self-tapping screws is shown at the rigid frame connection in Fig. 1. The installation of the long and very thin screws brings about challenges with regard to the precise positioning inside the timber section. Due to the fiber direction of the material or inhomogeneities such as knots or cracks, the screws tend to stray from their designated axis. The increased deviations of longer screws can result in collisions within the wood or even exiting through the wood surface, thus reducing the load bearing capacity of the reinforcement or joint. 


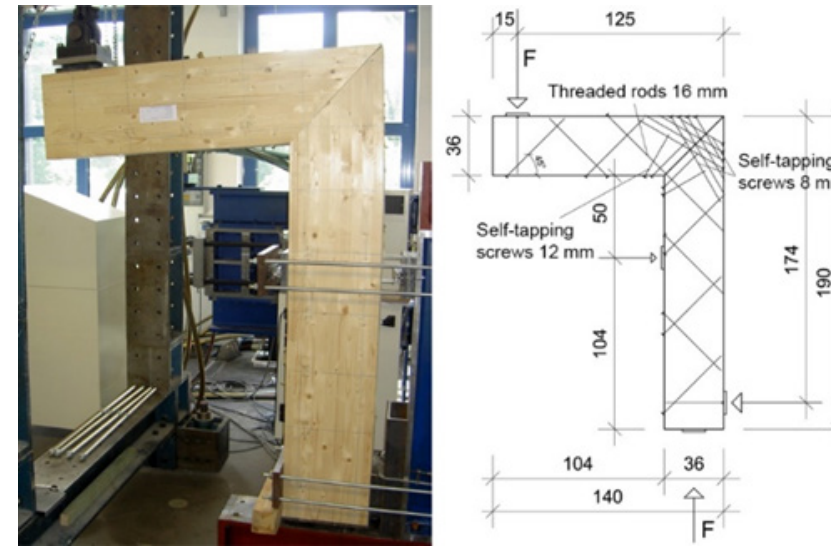

Fig. 1 Rigid frame corner joined with self-tapping screws. (Dimensions in $\mathrm{cm}$ ) [18]

The existence of a guide hole with a diameter close to the core diameter of the screw significantly improves the precise positioning of long self-tapping screws. Nevertheless, a conventional mechanical drilling leads to similar deviations as the thin drill behaves similar to the screws itself. Thus, pre-drilling holes by means of laser radiation shows promise as an innovative method to produce straight guide holes [20].

The topic of this paper is the investigations on machining wood using a high power USP-laser source. Firstly, a report is drawn up covering the fundamental investigations of the optical characteristics of wood, as well as the USP ablation of wood. Afterwards, the initial results are presented, which demonstrate how the full potential of a high power USP laser source can be applied to the deep drilling of structural timber (with slight thermal degeneration being tolerated). Moreover, a pullout test is used to investigate the influence of the laser pre-drilled borehole and the heat affected zone on the mechanical strength of the screw connection".

\section{The material wood and glulam}

Wood is an inhomogeneous, naturally growing material, which is highly anisotropic and porous. Therefore, all properties of this material are subject to strong variations and dependent on numerous influences. The physical properties such as mechanical strength and optical properties are mainly characterized by the macro- and microstructure of wood, yet they also depend on the chemical composition. In the commercial field, wood is distinguished between hardwood and softwood which are obtained from angiosperms and gymnosperms from a botanical point of view, respectively [21]. Within this work only spruce wood is processed which belongs to the softwoods. In temperate climate zones, wood cell production of trees changes seasonal and annual rings with different cell densities are formed [21]. Microstructural, softwood consists of up to $90-95 \%$ of tracheid cells $[21,22]$ which are elongated cells along the growth direction and are also called fibers [21]. Due to the cell geometry tracheid cells have a light conducting property in the longitudinal direction which is called "tracheid-effect" [23]. From a chemical point of view, these structures consist to 40 $-50 \%$ of cellulose, $25-35 \%$ of hemicellulose, $18-35 \%$ of lignin and $4-10 \%$ of inorganic minerals [24]. Cellulose, hemicellulose and lignin are bio polymers. Because of these bio polymers, the melting of laser treated wood surfaces is possible $[10,15]$.

Having these macro- and microscopic structures in mind, it becomes more evident that the properties of wood are subject to strong statistical fluctuations. Therefore, the reported irregularity of wood ablation can be attributed to the wood structure itself. Its fluctuating density originates from the fiber structure and growth ring structure with alternating early and late wood [10]. This density difference between early and late wood was derived by Eder et al. [25] for a Norway spruce and amounts approximately to a factor of 5 . Thus, reproducible laser processing of wood becomes a very challenging task. Furthermore, the anisotropy of the material properties such as the thermal behavior due to the fiber structure may influence the laser material interaction. However, the thermal conductivity of spruce wood parallel to the fiber direction is approximately factor two higher as perpendicular to the fiber, which is a low anisotropy factor compared to other fiber based materials. For example unidirectional carbon-fiber-reinforced polymers have a anisotropy factor of over 100 [26].

In order to reduce the fluctuations and improve specific properties, many wood-based materials such as glued laminated timber (glulam) have been developed. Glulam consists of multiple lamellas of wood, which are glued fiber parallel with each other. Thus, the strength of glulam can be increased up to $10 \%$ compared to the raw wood and fluctuation of properties is decreased significantly [22]. In this work, glulam according to DIN EN 14080:2013 [27] with strength class GL24h, an average gross density of the lamellas of $425 \mathrm{~kg} / \mathrm{m}^{3}$, a melamine based gluing and a lamella thickness of $30 \mathrm{~mm}$, is utilized for the experiments.

\section{Fundamental investigation of wood laser ablation}

In order to gain a deeper insight of the interaction between ultrashort pulses and wood, fundamental investigations are carried out. Firstly, a detailed analysis of the optical characteristics of timber is conducted for the applied wavelength of the USP-laser. Secondly, the ablation behaviour of timber is investigated by creating cavities on the surface with varied process parameters.

\subsection{Experimental setup and procedure}

The optical characteristics are studied with an UV/Vis/NIR spectrophotometer LAMBDA 1050+ by PerkinElmer. Measurements are performed for the two main components of timber cellulose and lignin, separated early and late wood and glulam itself.

The fundamental ablation experiments in this work are carried out with an Amphos 400 ultrashort pulsed high power laser system. The emitted laser radiation is focused with an f-theta objective "JENarTM 160-1030...1080-110" from JENOPTIK Optical Systems GmbH with a focal length of $160 \mathrm{~mm}$. For the beam deflection an excelliSCAN 14 from Scanlab $\mathrm{GmbH}$ is utilized. The spot diameter on the sample surface is $2 \mathrm{w}_{0}=60 \mu \mathrm{m}\left(1 / \mathrm{e}^{2}\right)$. The laser system is capable of emitting pulses with variable pulse durations between $\tau=2-20$ ps at a central wavelength of $\lambda=1030 \mathrm{~nm}$. In this work, the pulse duration for all investigations is set to the shortest value of $\tau=2 \mathrm{ps}$. The repetition rate is adjustable between $\mathrm{f}_{\text {rep }}=0.4-40 \mathrm{MHz}$ with an average power of 
$\bar{P}=340 \mathrm{~W}$. Lower repetition rates are obtained by an integrated pulse picker unit. The achieved ablation volume and surface morphology is evaluated with a digital microscope (Keyence VHX-6000).

\subsection{Optical characteristics of timber}

In general, wood possesses an absorption spectrum which is similar to water with high absorptance ( $\mathrm{A} \cong 90 \%$ ) for UV- and IR-radiation and a minimum at a wavelength of about $1 \mu \mathrm{m}[12,28]$. Depending on the wood type, the absorptance at this wavelength is typically in the range between $10-20 \%[28,29]$. For this reason, most of the wood processing with laser radiation was done with $\mathrm{CO}_{2}$-lasers in the past. However, ultrafast laser systems which are typically used for material processing have a wavelength of $1 \mu \mathrm{m}$. Therefore, only a small fraction of the pulse energy $E_{P}$ will be coupled into the material, omitting non-linear effects. On the other hand, the aforementioned "tracheid-effect" might enhance the coupling of the pulse energy into the wood if the incoming beam is parallel to the tracheid fibers. Therefore, the tracheid effect could be beneficial for the deep drilling of timber. For very high intensities also nonlinear effects like multi-photon ionization can lead to plasma generation and to an increased absorptance in wood eventually.

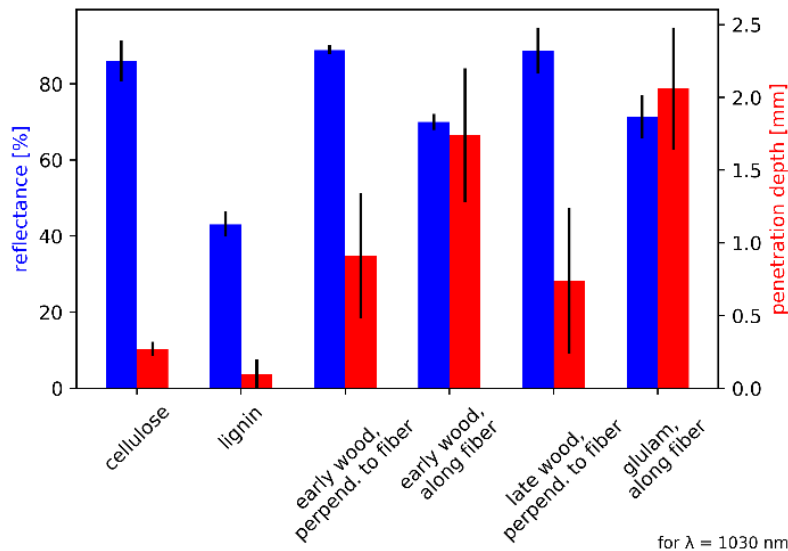

Fig. 2 Overview of the reflectance and optical penetration depth (of different components) of timber for $\lambda=1030 \mathrm{~nm}$.

In Fig. 2, the reflectance and penetration depth of the two main components of wood and early and late wood for different orientations of the tracheid fibers are shown. The reflectance is measured directly via the spectrophotometer, while the penetration depth is calculated with the LambertBeer law by measured transmission values for samples of different thickness by $\delta=-d \cdot \ln ^{-1}\left(\frac{I(d)}{I_{0}}\right)$, with the optical penetration depth $\delta$, sample thickness $d$ and the corresponding intensities I. The calculations are carried out for multiple samples and averaged whereas the standard deviation is represented by the error bars. Most measurements are performed on multiple samples to obtain an estimation for the variance of the acquired values because of the naturally present strong variations of material parameters. Cellulose has a reflectance similar to the reflectance of timber perpendicular to the fiber direction with a value close to $90 \%$. Lignin, on the other hand, possesses a reflectance of $43.1 \%$, making it the component with the lowest reflectance. Both of the isolated components also display a small penetration depth compared to the investigated timber material. The transmittance of light in wood is therefore a consequence of the wood structure. Late and early wood illuminated perpendicular to the fiber direction both exhibit a similar reflectance close to $90 \%$ and a penetration depth slightly below $1 \mathrm{~mm}$. The measured values for early wood along the fiber direction (measurements of solely late wood were not possible due to the low late wood thickness) show a significantly lower reflectance $(\mathrm{R} \cong 70 \%)$ with a higher penetration depth in the range of $\sim 2 \mathrm{~mm}$. In glulam, the presence of small late wood layers probably slightly increases the reflectance and decreases the penetration depth.

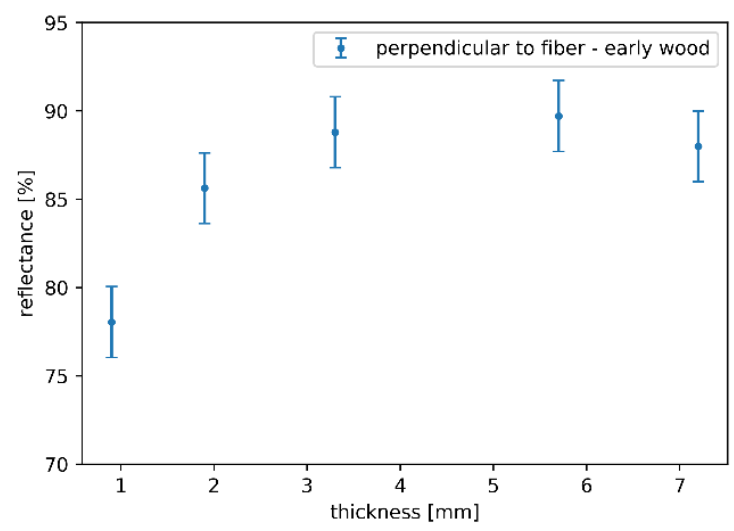

Fig. 3 Measured reflectance of each sample plotted as a function of its thicknesses perpendicular to the fiber direction for early wood for $\lambda=1030 \mathrm{~nm}$.

The reflectance as a function of thickness, measured perpendicular to the fibers, shows an unexpected behaviour as shown in Fig. 3. For thicknesses up to $3 \mathrm{~mm}$, the reflectance seems to increase with an increasing thickness. The reflectance for the samples perpendicular to the fiber direction shows an increase of reflectance with increasing sample thickness. One explanation is the back scattering of light from deeper layers of the sample. In this case, a portion of the reflected light first enters the material, is reflected inside the material and finally escapes again. The reflection presumably occurs at the walls of the tracheid cells.

\subsection{Experimental ablation results and discussion}

First of all, the ablation efficiency and ablation rate of glulam was investigated. For this purpose, cavities of the size of $3 \times 10 \mathrm{~mm}^{2}$ were ablated on the wood. All glulam ablation processes are carried out on samples where the wood fibers are oriented $90^{\circ}$ to the surface normal (wood fiber angle). The used process parameter listed in Table 1 are kept constant. Only the single pulse peak fluence $\left(F_{0}=\frac{2 E_{p}}{\pi w_{0}^{2}}\right)$, which is called single pulse fluence in the following, was varied. The number of passes was adapted to keep deposited energy constant. For the structuring process, a scanning strategy, with overlapping scan lines, is applied whereby the lines are scanned bidirectional and the planes (number of passes $n$ ) are alternately rotated by 90 degrees. The pulse overlap at constant repetition rate $\mathrm{f}_{\text {rep }}$ is defined by the scan velocity $\mathrm{v}_{\mathrm{s}}$. The line overlap is set via the line pitch $\Delta \mathrm{y}$. Table 1 Summary of the used parameter set for the ablation efficiency study of glulam 


\begin{tabular}{ll}
\hline process parameter & value \\
\hline wavelength $\lambda$ & $1030 \mathrm{~nm}$ \\
focus diameter 2w0 & $60 \mu \mathrm{m}$ \\
pulse duration $\tau$ & $2 \mathrm{ps}$ \\
scan speed $\mathrm{v}_{\mathrm{s}}$ & $0.3 \mathrm{~m} / \mathrm{s}$ \\
line pitch $\Delta \mathrm{y}$ & $30 \mu \mathrm{m}$ \\
repetition rate $\mathrm{f}_{\text {rep }}$ & $10 \mathrm{kHz}$ \\
single pulse fluence $\mathrm{F}_{0}$ & approx. $1-55 \mathrm{~J} / \mathrm{cm}^{2}$ \\
number of passes $\mathrm{n}$ & $76-1$ \\
wood fiber angle & $90^{\circ}$ \\
\hline
\end{tabular}

The ablated cavities are measured with the aforementioned VHX-6000. Due to the pronounced annual ring structure of the spruce wood used for the glulam, different ablation depths for early and late wood are observed (compare Fig. 4). A smooth transition between early wood (spring wood) and late wood (summer wood) can be measured in growth direction whereas the transition from late to early wood is steep. This corresponds remarkably with the gradual decrease of the cell size within an annual ring measured by Eder et al. [25]. The ablation depth for determination of the ablation efficiency and ablation rate is measured at the lowest point of the early wood and the highest point of the late wood ablation.

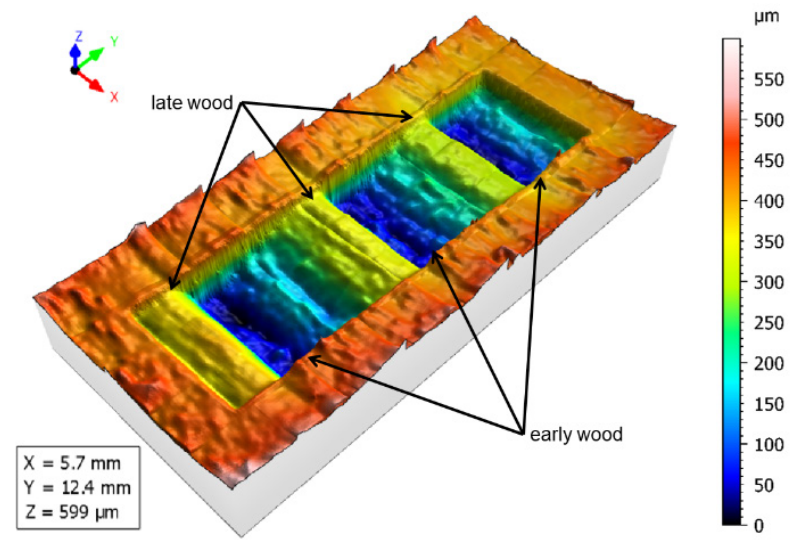

Fig. 4 Example of an ablated cavity on glulam surface. The different ablation due to the annual rings is visible.

The derived ablation efficiency is shown in Fig. 5 and the corresponding ablation rate is shown in Fig. 6. Due to the inhomogeneity of wood, the standard deviation of the present sample of measurements is up to $100 \%$ for single measuring points. However, for the ablation efficiency a trend can be recognized. After the maximum of approx. $20.6 \mathrm{~mm} / \mathrm{minW}$ for early wood is reached for $5 \mathrm{~J} / \mathrm{cm}^{2}$, the efficiency decreases. For the ablation efficiency of late wood, no clearly identifiable maximum occurs due to high fluctuation of the values. The corresponding efficiency of late wood for $5 \mathrm{~J} / \mathrm{cm}^{2}$ is $4.7 \mathrm{~mm}^{3} / \mathrm{minW}$. In between the measuring points for 32 and $34 \mathrm{~J} / \mathrm{cm}^{2}$ an offset to higher efficiencies is visible. This can be attributed to a change of the samples. For the ablation rate, on the other hand side, an almost linear progression up to $18 \mathrm{~J} / \mathrm{cm}^{2}$ can be recognized. Above this fluence a slight weakening in the course could be evident, which suggests shielding through increased ablation products. Similar to the ablation efficiency the change of samples is reflected by an offset in the measured values. The difference between early wood and late wood in its ablation behavior differs on average by a factor of 4.7. This corresponds well with the difference in density of early and late wood with a factor of approximately 5 derived by Edler et al. [25]. This indicates a direct relationship between the ablation behavior and the local density or the cell density of wood. Therefore, the reached ablation rates for the low density early wood are higher than the corresponding values for late wood with greater density.

For all measurements shown in Fig. 5 and Fig. 6 with exception of the ablation with the highest fluence, no discoloration of the surface and, according to this, no carbonization occurs. Therefore, an ablation rate improvement by increasing the fluence disregarding the efficiency with nearly constant surface quality seems possible within certain limits.

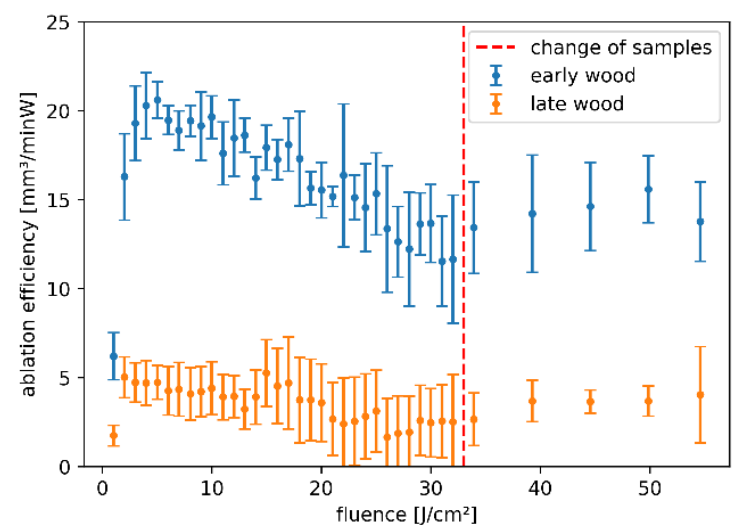

Fig. 5 Ablation efficiency of glulam (spruce wood) as a function of the single pulse fluence.

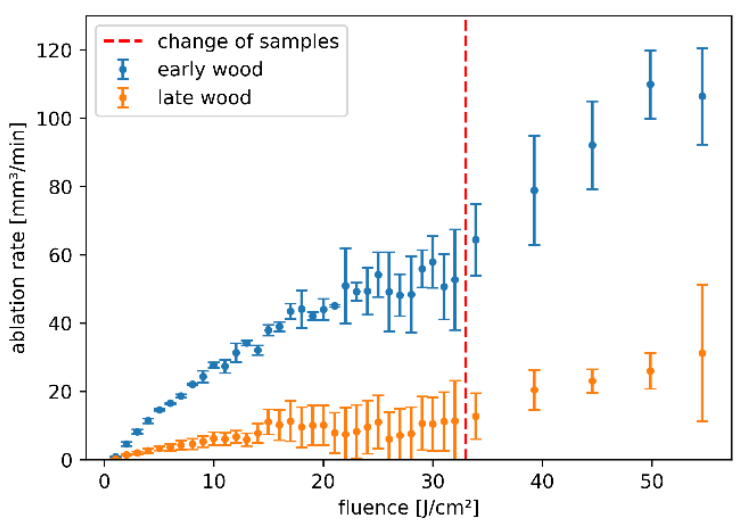

Fig. 6 Ablation rate of glulam (spruce wood) as a function of the single pulse fluence.

For a further comparison of the early and late wood ablation behavior the ablation threshold was determined. Due to the rough wood surface (cf. Fig. 4), no direct method for the determination of the ablation threshold such as introduced by Liu [30] could be applied. Instead, the effective energy penetration depth and ablation threshold fluence was determined via a fit method of the ablation efficiency. For this method the theoretical dependence of the ablation efficiency is descripted by $\varepsilon=\frac{1}{2} \frac{\delta_{E}}{F_{0}} \ln ^{2}\left(\frac{F_{0}}{F_{t h}}\right)[31,32]$ based on an USP ablation model by Nolte et al. [33]. Where $\varepsilon$ is the ablation efficiency, $\delta_{\mathrm{E}}$ the energy penetration depth, $\mathrm{F}_{0}$ the single pulse fluence and $F_{\text {th }}$ the threshold fluence for ablation. A detailed derivation of this formula can be found elsewhere 
[34]. For the following fits (Fig. 7) only the data of the measurement series carried out on the same sample is used.

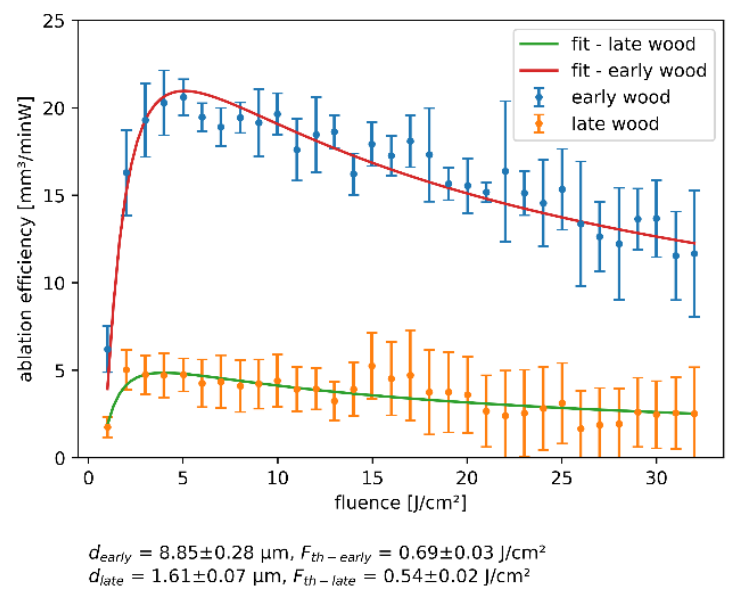

Fig. 7 Fit for the ablation efficiency of early and late wood for glulam (spruce wood) with derived energy penetration depth and ablation threshold.

From these fits the ablation threshold and energy penetration depth is derived to $F_{\text {th-early }}=0.69 \mathrm{~J} / \mathrm{cm}^{2}$ and $\mathrm{d}_{\text {early }}=8.85 \mu \mathrm{m}$ for early and $\mathrm{F}_{\text {th-late }}=0.54 \mathrm{~J} / \mathrm{cm}^{2}$ and $\mathrm{d}_{\text {late }}=1.61 \mu \mathrm{m}$ for late wood. According to these values the threshold fluence for which an ablation of the wood surface becomes evident is similar for early and late wood. Therefore, the different ablation efficiency is attributable to the different energy penetration depth, which differ similar to the ablation rate by a factor of 5.5. This leads to the hypothesis that the ablation of early and late wood is a similar process and depends in first order on the difference in the density of cells. In other words, the ablation processes within the same type of wood yields a constant mass ablation rate but due to the different density caused by the wood structure the volume ablation rate varies.

The energy penetration depths derived from the fits are two orders of magnitude below the measured optical penetration depths for the UV-VIS measurement shown in Fig. 3. Considering only linear absorption and no additional energy transport the values should match. The observed difference may be due to non-linear absorption effects within the USP ablation process or the values are not comparable due to not fully understood optical properties of the biological material (e.g. tracheid effect). Considering the difference in intensity in the order of magnitudes of the applied light for the UVVIS measurement and the focused ultrashort pulsed laser the occurrence of non-linear absorption effects is expected. Furthermore, the light guiding effects of the cell structure will presumably be disturbed during the ablation process which enhances a surface-near energy deposition. Because of these effects the values cannot be compared directly.

Since wood is a thermally sensitive material and tends to carbonize and oxidize, the first experiments are carried out with a repetition rate of $10 \mathrm{kHz}$. Because of the low repetition rate, carbonization is only observed for very high single pulse fluences. In the following, the possibility of increasing the productivity by scaling the repetition rate is investigated. The repetition rate is varied between 10 to $400 \mathrm{kHz}$ and the scan speed is adapted to achieve a constant pulse overlap of $50 \%$ of the focus diameter. The single pulse fluence was set to $5 \mathrm{~J} / \mathrm{cm}^{2}$ where the maximum ablation efficiency is achieved (cf. Fig. 5). An overview of all used process parameters is shown in Table 2.

Table 2 Summary of the used parameter set for the ablation rate scaling via the repetition rate.

\begin{tabular}{ll}
\hline process parameter & value \\
\hline wavelength $\lambda$ & $1030 \mathrm{~nm}$ \\
focus diameter $2 \mathrm{w} 0$ & $60 \mu \mathrm{m}$ \\
pulse duration $\tau$ & $2 \mathrm{ps}$ \\
scan speed $\mathrm{v}_{\mathrm{s}}$ & $0.3-12 \mathrm{~m} / \mathrm{s}$ \\
line pitch $\Delta \mathrm{y}$ & $30 \mu \mathrm{m}$ \\
repetition rate $\mathrm{f}_{\text {rep }}$ & $10-400 \mathrm{kHz}$ \\
single pulse fluence $\mathrm{F}_{0}$ & approx. $5 \mathrm{~J} / \mathrm{cm}^{2}$ \\
number of passes $\mathrm{n}$ & 15 \\
wood fiber angle & $90^{\circ}$ \\
\hline
\end{tabular}

Analogous to the previous evaluation the ablation efficiency and ablation rate is determined separately for early and late wood and is visualized in Fig. 8 as a function of the repetition rate. The ablation efficiency remains constant for all investigated repetition rates within the scope of the measuring accuracy. According to the course of the efficiency an almost linear behavior is observed for the ablation rate due to the higher repetition rate as also seen in the lower graph in Fig. 8. This results in a maximum ablation rate of $630 \mathrm{~mm}^{3} / \mathrm{min}$ for early and $107 \mathrm{~mm}^{3} / \mathrm{min}$ for late wood utilizing a repetition rate of $400 \mathrm{kHz}$. This is an increase of a factor 43.6 and 40.9 compared to the process with $10 \mathrm{kHz}$, respectively. Based on these results, no significant inter pulse interactions as heat accumulation or shielding effects are present. A possible explanation is that the shielding effect is not yet pronounced enough due to the low repetition rates. Heat accumulation, on the other hand, has probably not started within the measurement series because no significant deviation from the linear increase of the ablation rate occurred. Furthermore, no visible thermal degeneration of the processed area is evident. Therefore, the anisotropy of the thermal properties $-0.22-0.33 \mathrm{~W} / \mathrm{mK}$ parallel to the fiber and $0.09-0.12 \mathrm{~W} / \mathrm{mK}$ perpendicular to the fiber [22]- of a factor 2 in turn has no significant influence either.

For a qualitative evaluation the microscopic image of the processed surface is shown in Fig. 9. No discoloration and carbonization is visible on the processed surface. Furthermore, no significant difference of the surface morphology becomes evident for increasing repetition rates. Therefore, an increase of the productivity of wood structuring processes via repetition rate scaling is a promising approach. The limits for this approach have yet to be determined. 

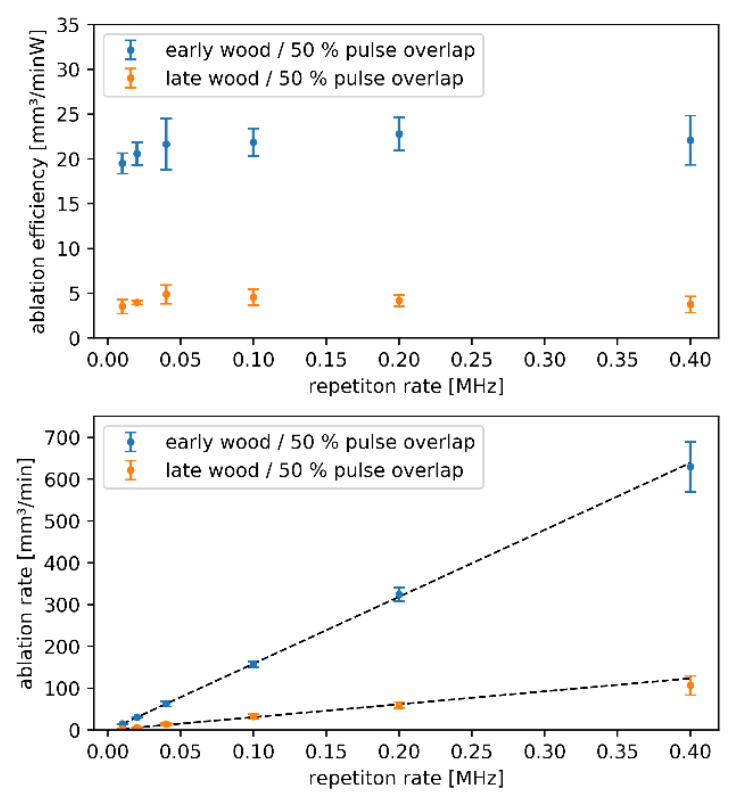

Fig. 8 Ablation efficiency and ablation rate of glulam (spruce wood) as a function of the pulse repetition rate with constant single pulse fluence and pulse overlap.

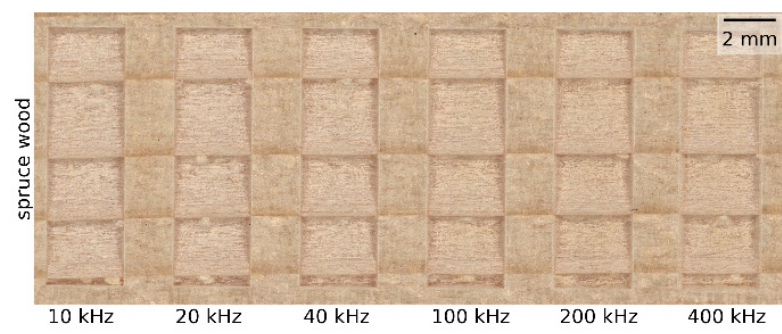

Fig. 9 Microscope image of the structuring series on spruce wood with increasing repetition rate. No discoloration or carbonation is visible.

\section{Deep drilling glulam}

Based on the above findings on the USP ablation of spruce wood deep drilling processes are designed for glulam. In the following, a short overview of the ongoing research is presented.

\subsection{Experimental setup and procedure}

The USP deep drilling experiments in this work are carried out with same Amphos 400 high power laser source as described in section 3.1. The scanner system from Scanlab $\mathrm{GmbH}$ was also utilized for beam deflection. Since holes with a diameter of about $5 \mathrm{~mm}$ (approx. core diameter of an $8 \mathrm{~mm}$ STS) should be created in samples with up to $300 \mathrm{~mm}$ thickness, the optical setup is adapted to be able to shift the focal plane into the hole. This is only possible without significant clipping at the borehole entrance if the deflection angle of the beam is higher than the divergence angle of the convergent beam, see Fig. 10. If this condition is not fulfilled, a fraction of the convergent beam is shielded at the borehole entry. Therefore, a plan convex lens with a focal length of $500 \mathrm{~mm}$ is positioned before the scanner. With a raw beam diameter of about $5 \mathrm{~mm}$, the above mentioned condition is fulfilled with this optical setup underneath the scanner. The existing imaging error due to the missing plane field optic can be neglected because of the small deflection angles. Additionally, this results in a fluence close to the optimum fluence for the maximum pulse energy of the laser source, which increases the ablation rate at a given repetition rate.

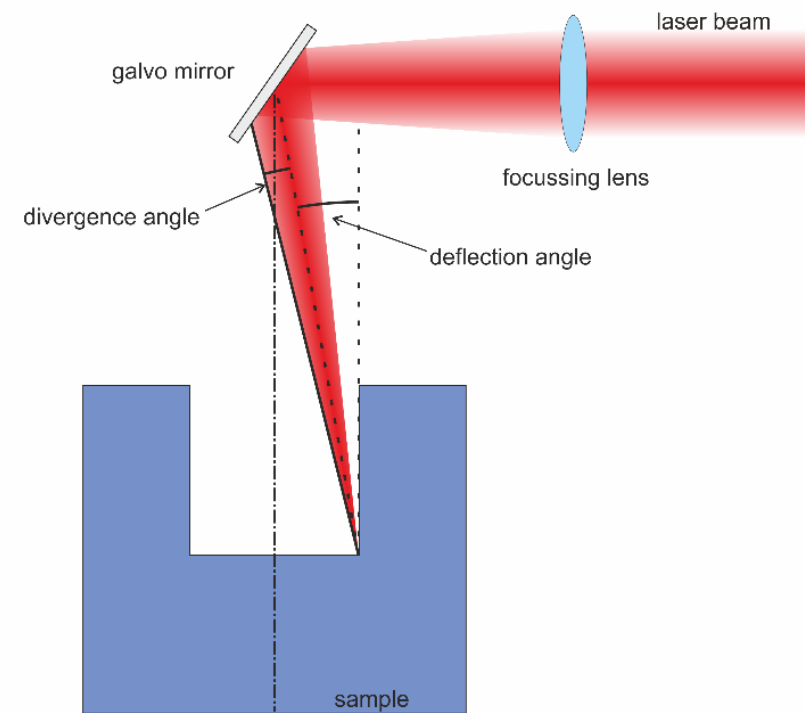

Fig. 10 Schematic illustration of the drilling optics design for the beam tilt with a plan convex lens $(f=500 \mathrm{~mm})$ installed in front of the scanner mirrors.

The evaluation of the drilled holes is performed by the creation of a longitudinal section of the glulam sample. This allows the measuring of the local borehole diameter and the evaluation of the carbonization of the borehole wall.

\subsection{Experimental results and discussion}

For all investigated deep drilling processes the parameter set in Table 3 is used. Due to the other focusing optic the focus diameter has changed to $180 \mu \mathrm{m}$ and the fluence is set to $6.7 \mathrm{~J} / \mathrm{cm}^{2}$. Thus, the fluence is in the regime of the maximum ablation efficiency of the preliminary ablation tests and the maximum pulse energy $(850 \mu \mathrm{J})$ of the laser system is put into effect. For the scanning strategy spiral scan paths with a spiral pitch $\Delta \mathrm{r}$ of $100 \mu \mathrm{m}$ and the desired outer diameter are generated. Each spiral is rotated with respect to the previous one to obtain an equally ablated borehole area. The repetition rate is set to $50 \mathrm{kHz}$ since it is desirable to choose it as high as possible for a productive process but due to the hot ablation products within the deep borehole with rates above $50 \mathrm{kHz}$ incineration took place. For $50 \mathrm{kHz}$ still a carbonization of the borehole wall happens which results in a widening of the borehole diameter. However, to minimize the oxidation, the borehole entry is exposed to nitrogen to keep the oxygen in the atmosphere away from the ablation zone. In order to maintain the focus of the beam at the bottom of the borehole a feed speed $\mathrm{v}_{\mathrm{z}}$ was adjusted via parameter studies. Because of a high Rayleigh length $(\sim 24 \mathrm{~mm})$, deviations between focal plane and borehole ground have only a minor effect on the ablation rate. Furthermore, the drilling process must be periodically paused otherwise the process will eventually come to a standstill at some point. This presumably results from the increasing interaction of the laser beam with the ablation products within the borehole since it becomes more evident with advancing depth. For this purpose a step width of $5 \mathrm{~mm}$ and a subsequent pause of 
approximately $5 \mathrm{~s}$ has proven sufficient to clear the bore hole from most of the ablation products.

Table 3 Summary of the used parameter set for the deep drilling processes.

\begin{tabular}{ll}
\hline process parameter & value \\
\hline wavelength $\lambda$ & $1030 \mathrm{~nm}$ \\
focus diameter $2 \mathrm{w} 0$ & $180 \mu \mathrm{m}$ \\
pulse duration $\tau$ & $2 \mathrm{ps}$ \\
scan speed $\mathrm{v}_{\mathrm{s}}$ & $5 \mathrm{~m} / \mathrm{s}$ \\
spiral pitch $\Delta \mathrm{r}$ & $100 \mu \mathrm{m}$ \\
offset angle & $108^{\circ}$ \\
repetition rate $\mathrm{f}_{\text {rep }}$ & $50 \mathrm{kHz}$ \\
single pulse fluence $\mathrm{F}_{0}$ & approx. $6.7 \mathrm{~J} / \mathrm{cm}^{2}$ \\
feed speed $\mathrm{v}_{\mathrm{z}}$ & $0.3-1 \mathrm{~mm} / \mathrm{s}$ \\
wood fiber angle & $0^{\circ} ; 30^{\circ} ; 60^{\circ} ; 90^{\circ}$ \\
\hline
\end{tabular}

For the deep drilling experiments glulam samples with varied thickness and four fiber angels $0^{\circ}, 30^{\circ}, 60^{\circ}$ and $90^{\circ}$ are used. The achievable feed speed and therefore the process time varied with the fiber angle. For a $90^{\circ}$ fiber direction the slowest feed speed is necessary and thus these processes will take the most time. The feed speed can be scaled linear to the fiber direction up to a maximum speed of $1 \mathrm{~mm} / \mathrm{s}$ for $0^{\circ}$ fiber angle. Furthermore, the achieved outer diameter varies within the borehole due to the inhomogeneities of the material. This must be taken into account while designing a deep drilling process. However, for all fiber directions $160 \mathrm{~mm}$ thick glulam is successfully drilled through with a maximum local diameter of $5 \mathrm{~mm}$. Furthermore, as shown in Fig. 11, even knotholes and changes of the earlylate wood density within the glulam can be compensated by a suitable process design.

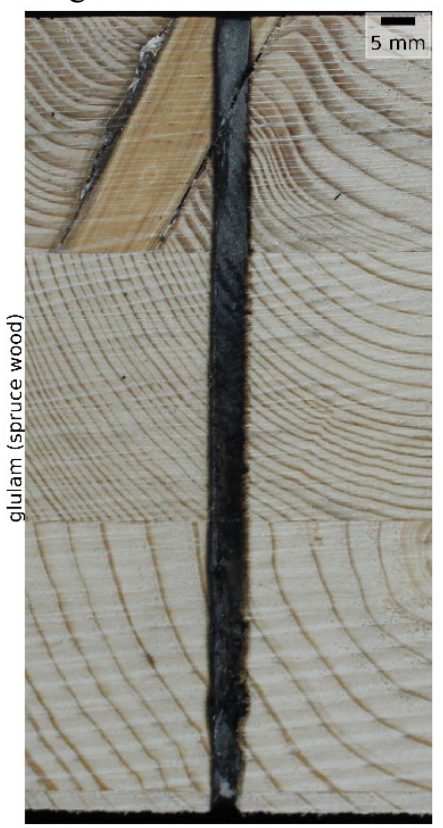

Fig. 11 Cross section of a sample for USP deep drilling with knothole within $120 \mathrm{~mm}$ thick glulam. The carbonized borehole wall and the density changes in the lamellas are visible.

\subsection{Mechanical tests}

The effect of USP laser pre-drilling on the displacement of the STS is evaluated by measuring the offset of the exit of single screws inserted in glulam samples of different lengths with respect to their designated axes. Hereby, the screws inserted without pre-drilling show deviations of up to $7 \mathrm{~mm}$ for an embedding length of $120 \mathrm{~mm}$. These deviations increase with higher embedding lengths. The screws inserted in pre-drilled holes follows the axis and no deviations occurs, as long as the pre-drilling is conducted through the whole length of the samples.

Furthermore, the load-bearing behavior of single screws in laser pre-drilled guide holes is evaluated on the basis of the characteristic of the load displacement curve and the withdrawal resistance determined by pull-out tests. The results are compared to the results of not pre-drilled screw connections. Fig. 12 shows the load-displacement-curves for screws with a diameter of $8 \mathrm{~mm}$, inserted parallel to the fiber direction $\left(\alpha=0^{\circ}\right)$, with an embedding length of $120 \mathrm{~mm}$.

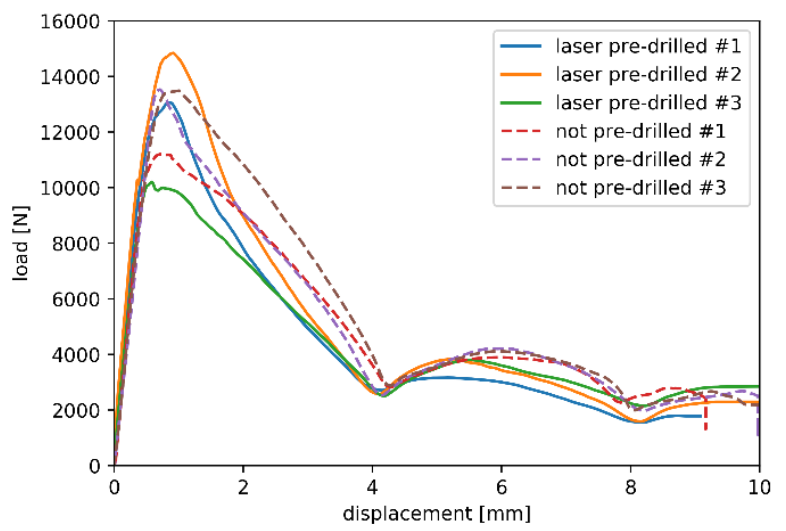

Fig. 12 Load-displacement-curve for $120 \mathrm{~mm}$ glulam (fiber angle $0^{\circ}$, self-tapping screws with $\mathrm{d}=8 \mathrm{~mm}$ ).

In general, the screws show a similar load bearing characteristic. Only the range beyond the maximum shows a steeper drop, which indicates a changed friction behavior of the laser pre-drilled screw connections.

When comparing the mean withdrawal resistances there is no negative effect derived from heat affected zones by the laser treatment visible. The mean withdrawal resistance is not significantly different from the screw connections without pre-drilling.

\section{Conclusion}

Deep drilling of wood has been investigated towards the use for pre-drilling of connection screws in rigid timber wood constructions.

The optical properties of glulam, cellulose and lignin were investigated via UV-VIS measurements. The results show that the ablation process is most likely initiated by absorption within lignin between the wood fibers. Furthermore, a drop in reflectance and increase of optical penetration depth was measured for a change of the fiber direction of the sample from perpendicular to parallel to the incident light. This can be attributed to the reported tracheid effect in which the cells act as light guides [23]. Additionally, an increase of the reflectance perpendicular to the fiber was measured with increasing sample thickness which is attributed to a back scattering of light at cell walls from deeper layers of the sample. 
The fundamental ablation experiments show a different ablation behavior for early and late wood. By average this ratio yields 4.7 for the ablation rates. A sawtooth-like structure was observed in profiles of the processed samples due to the alternating annual rings. The ablation threshold for both types of wood are similar but the penetration depth differs by a factor 5.5. As this corresponds to the difference in density, we assume that the ablation of early and late wood is a similar process and depends in first order on the difference in the density of cells. In other words, the ablation processes within the same type of wood yields a constant mass ablation rate but due to the different density caused by the wood structure the volume ablation rate varies.

Those findings were converted into a deep drilling process for glulam. Deep drilling of structural timber with $160 \mathrm{~mm}$ thickness despite the random occurrence of knotholes and changing early late wood density was successfully carried out. No displacement occurred for the laser drilled boreholes. A carbonization of the borehole wall cannot be prevented although nitrogen is applied to the borehole entry. This is attributed to the hot ablation products within the borehole. To examine the influence of the heat affected zone and carbonization because of the laser pre-drilling, pull out test were performed. The load displacement behavior shows no difference in the maximum resistance compared with not pre-drilled screw connections. This indicates that the material damage by laser deep drilling with USP laser radiation has no negative effects on the mechanical connections realized.

In further investigations the USP laser ablation and deep drilling will be compared to utilization of other lasers sources in terms of efficiency, productivity, processing quality and influence on mechanical properties. Furthermore, the ablation products and the ablation surface of the USP processed spruce wood will be evaluated on a cellular and elementary level and compared to the ablation of other soft and hard woods.

\section{Acknowledgments and Appendixes}

The authors would like to thank the German Research Foundation DFG for funding this work under Grant No. 406036741

\section{References}

[1] C. W. McMillin and J. E. Harry: Forest Products Journal, (1971) 35.

[2] C. C. Peters and H. L. Marshall: U.S.D.A Forest Service Research Paper, (1975).

[3] G. Wieloch and P. Pohl, Proc. SPIE, Vol. 2202, (1995) 604

[4] R. Poprawe, C. Hinke, W. Meiners, F. Eibl, O. Zarei, M. Voshage, S. Ziegler, J. H. Schleifenbaum, A. Gasser, T. Schopphoven, E. Willenborg, J. Flemmer, C. Weingarten, J. Finger, and M. Reininghaus, Proc. SPIE, Vol. 10519, (2018) 6.

[5] M. N. Islam, K. Ando, H. Yamauchi, Y. Kobayashi, and N. Hattori: Wood Sci. Technol., 42, (2008) 343.

[6] M. N. Islam, K. Ando, H. Yamauchi, D. Kamikawa, T. Harada, and N. Hattori: BioResources, 8, (2012).

[7] Y. Wang, K. Ando, and N. Hattori: Wood Sci. Technol., 47, (2013) 447.
[8] S. Nath, D. G. Waugh, G. A. Ormondroyd, M. J. Spear, S. F. Curling, A. J. Pitman, and P. Mason: Lasers in Engineering (Old City Publishing), (2020) 381.

[9] Jerrold E. Winandy and Jeffrey J. Morrell: Wood and Fiber Science, 30, (2007) 185.

[10] M. Panzner, G. Wiedemann, K. Henneberg, R. Fischer, T. Wittke, and R. Dietsch: Appl. Surf. Sci., 127-129, (1998) 787.

[11] S. Fukuta, M. Nomura, T. Ikeda, M. Yoshizawa, M. Yamasaki, and Y. Sasaki: Holz als Roh-und Werkstoff, 74, (2016) 261.

[12] S. Fukuta, M. Nomura, T. Ikeda, M. Yoshizawa, M. Yamasaki, and Y. Sasaki: J. Wood Sci., 62, (2016) 316.

[13] C. Leone, V. Lopresto, and I. de Iorio: Optics and Lasers in Engineering, 47, (2009) 161.

[14] E. Baubeau, R. Le Harzic, C. Jonin, E. Audouard, S. Mottin, M. Courbon, and P. Laporte, Proc. SPIE, Vol. 4088, (2000) 48.

[15] F. Théberge, S. Petit, A. Iwasaki, M. R. Kasaai, and S.L. Chin: Appl. Surf. Sci., 191, (2002) 328.

[16] N. Naderi, S. Legacéy, and S.L. Chin: Forest Products Journal, 49, (1999) 73.

[17] S. Jakob, M. J. Pfeifenberger, A. Hohenwarter, and R. Pippan: Sci. Technol. Adv. Mater., 18, (2017) 574.

[18] M. Trautz and C. Koj: Bautechnik, 85, (2008) 190.

[19] M. Trautz and C. Koj: Bautechnik, 86, (2009) 228.

[20] M. Trautz, C. Koj, and H. Uchtmann: "Load bearing behaviour of self-tapping screws in laser-drilled guideholes" ed. by J. Eberhardsteiner, W. Winter, and A. Fadai, (2016) p. 238.

[21] R. A. Parham and R. L. Gray: "Formation and Structure of Wood" ed. by R. Rowell, (American Chemical Society, Washington, DC, 1984), Vol. 207 p. 3.

[22] P. Niemz and W. Sonderegger: Holzphysik, (Carl Hanser Verlag GmbH \& Co. KG, München, 2017).

[23] J. Nyström: Computers and Electronics in Agriculture, 41, (2003) 91.

[24] R. C. Pettersen: "The Chemical Composition of Wood" ed. by R. Rowell, (American Chemical Society, Washington, DC, 1984), Vol. 207 p. 57.

[25] M. Eder, K. Jungnikl, and I. Burgert: Trees, 23, (2009) 79.

[26] M. Kuribara and H. Nagano: Journal of Electronics Cooling and Thermal Control, 05, (2015) 15.

[27] DIN EN 14080:2013-09, Holzbauwerke Brettschichtholz und Balkenschichtholz Anforderungen; Deutsche Fassung EN_14080:2013, (Beuth Verlag GmbH, Berlin).

[28] P. Haller, E. Beyer, G. Wiedemann, M. Panzner, and H. Wust, Proc. 3rd Int. Symp. on Wood Machining., (2007) 183.

[29] H. Wust: Die Wirkung von Laserstrahlung auf strukturelle, chemische und physikalische Eigenschaften von Holz, (Shaker, Aachen, 2006).

[30] J. M. Liu: Opt. Lett., 7, (1982) 196.

[31] G. Raciukaitis: J. Laser Micro/Nanoengin., 4, (2009) 186.

[32] B. Jaeggi, B. Neuenschwander, M. Zimmermann, L. Penning, R. deLoor, K. Weingarten, and A. Oehler, Proc. SPIE, Vol. 8967, (2014) 89670Q. 
[33] S. Nolte, C. Momma, H. Jacobs, A. Tünnermann, B. N. Chichkov, B. Wellegehausen, and H. Welling: J. Opt. Soc. Am. B, 14, (1997) 2716.

[34] B. Bornschlegel, J. Köller, and J. Finger: J. Laser Micro/Nanoengin., 15, (2020) 56.

(Received: June 26, 2020, Accepted: October 13, 2020) 